\title{
Lidil
}

Revue de linguistique et de didactique des langues

\section{Prédicats et quasi-prédicats sémantiques dans une perspective lexicographique}

Igor Mel'čuk et Alain Polguère

\section{OpenEdition}

\section{Journals}

Édition électronique

URL : http://journals.openedition.org/lidil/2691

DOI : 10.4000/lidil.2691

ISSN : $1960-6052$

\section{Éditeur}

UGA Éditions/Université Grenoble Alpes

\section{Édition imprimée}

Date de publication : 1 juin 2008

Pagination : 99-114

ISBN : $978-2-84310-124-3$

ISSN : $1146-6480$

\section{Référence électronique}

Igor Mel'čuk et Alain Polguère, « Prédicats et quasi-prédicats sémantiques dans une perspective lexicographique », Lidil [En ligne], 37 | 2008, mis en ligne le 01 septembre 2009, consulté le 19 avril 2019. URL : http://journals.openedition.org/lidil/2691 ; DOI : 10.4000/lidil.2691

Ce document a été généré automatiquement le 19 avril 2019

(C) Lidil 


\title{
Prédicats et quasi-prédicats sémantiques dans une perspective lexicographique
}

\author{
Igor Mel'čuk et Alain Polguère
}

1 La notion de prédicat sémantique est bien établie en linguistique: on considère que certaines lexies (unités lexicales) comme comBATTRE, сомBAт, сомBATIF, etc. possèdent un sens prédicatif. Un sens prédicatif dénote un fait impliquant des participants qui correspondent aux arguments du prédicat en question. Nous reviendrons, dans la prochaine section, sur la notion linguistique de prédicat sémantique, qui est distincte de la notion logico-mathématique. Il suffit de mentionner pour l'instant que, traditionnellement, les sens prédicatifs sont opposés aux sens non prédicatifs, qui, eux, dénotent des entités. Tel est le cas, par exemple, des sens des lexies ÉTOILE, LION, CAILlOU, etc. Cependant, la distinction qui semble clairement établie entre sens prédicatifs et sens non prédicatifs masque en fait la présence d'un ensemble très intéressant, et très important en langue, de sens « intermédiaires », qui tiennent à la fois des prédicats et des non-prédicats. Comme les non-prédicats, ces sens dénotent des entités, et non des faits. Pourtant, comme les prédicats, ils ne peuvent être modélisés sans tenir compte de «positions sémantiques » qu'ils contrôlent, positions que l'on peut représenter par des variables (X, Y, Z...). Il s'agit, par exemple, du sens des noms comme MINISTRE ' $[\mathrm{X}]$ ministre responsable de $\mathrm{Y}$ dans le gouvernement du pays Z', PARTIE '[X] partie de $\mathrm{Y}$ ' (comme dans une partie de la maison), HôPITAL 'hôpital où $\mathrm{X}$ soigne $\mathrm{Y}$ ', etc. Nous appelons quasi-prédicats ces sens hybrides. L'ensemble des quasi-prédicats d'une langue est très hétérogène, et chaque type de quasi-prédicat pose ses propres problèmes de modélisation. Nous proposons ici une analyse des différents types de quasi-prédicats présents dans les langues, en adoptant une perspective lexicographique. Plus précisément, nous nous situons dans le cadre de la Lexicologie Explicative et Combinatoire (Mel'čuk et al., 1995), en empruntant nombre de nos illustrations aux données de la base lexicale DiCo des dérivations sémantiques et collocations du français (Polguère, 2000 ; Mel'čuk et Polguère, 2006) et au Lexique actif du français ou LAF (Mel'čuk et Polguère, 2007). 
2 Nous commencerons par préciser la notion de prédicat sémantique. Cela nous amènera à traiter la notion d'actant, qui lui est intimement liée. Dans la section suivante, nous traiterons $\mathrm{du}$ cas très particulier des prédicats non actanciels. Finalement nous présenterons les quasi-prédicats, en offrant notamment une caractérisation de plusieurs types de quasi-prédicats qui abondent dans les lexiques des langues naturelles.

\section{La notion de prédicat sémantique}

3 La grande majorité des sens lexicaux des langues naturelles possèdent la propriété d'être des sens liants (Polguère, 1992 : 144ssq, 1997 : 3-4). Un sens liant typique dénote un fait: quelque chose dont on peut dire que cela a ou n'a pas lieu. Un fait peut être un évènement, une action, une activité, un état, un processus, une propriété, une relation, une quantité, etc. Un fait présuppose nécessairement un certain nombre de participants ; pour cette raison, il est approprié de modéliser les sens liants par des micro-structures sémantiques qui contiennent la représentation des participants potentiels des faits en question. Dans notre approche, cette micro-structure peut être visualisée sous la forme d'un graphe du type réseau sémantique constitué du sens liant lui-même, connecté aux variables qui désignent les "positions disponibles" pour les sens correspondant aux participants. Ce type de structure est illustré dans la figure ci-dessous, à partir du sémantème - c'est-à-dire, du signifié - du verbe ÉLIRE [Nous avons élu Marie-Claude présidente pour cinq ans].

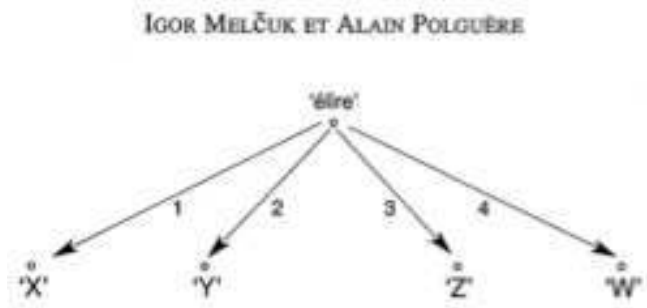

Figure 1 - 'X élit l'individu Y au poste Z pour la période W'

4 plus, nous ferons usage dans ce qui va suivre de la terminologie suivante :

- sens prédicatif, ou prédicat, désignera un sémantème liant qui dénote un fait ('élire', 'élection', 'éligible', 'pendant [les élections]'...);

- actant sémantique d'un prédicat dans une phrase donnée désignera un sens qui correspond à un participant du fait dénoté par le prédicat en question et qui est exprimable dans la phrase auprès de ce prédicat ;

- structure actancielle désignera la micro-structure sémantique associée au prédicat dans le lexique ;

- forme propositionnelle désignera la représentation d'une structure actancielle; la forme propositionnelle peut être soit une expression linéaire de nature linguistique, comme dans le titre de la figure 1, soit un réseau sémantique, comme dans la figure 1 elle-même. métaphore scientifique construite à partir de la notion de prédicat logique. Un prédicat logique $\mathrm{p}$ dénote une propriété ou une relation et permet de construire une fonction propositionnelle logique $\mathrm{p}(\mathrm{x}), \mathrm{p}(\mathrm{x}, \mathrm{y}), \mathrm{p}(\mathrm{x}, \mathrm{y}, \mathrm{z})$ etc., qui sera vraie pour tous les $\mathrm{x}, \mathrm{y}, \mathrm{z}$... compatibles avec la propriété ou relation $\mathrm{p}$. On voit le lien évident entre les notions de 
prédicat logique et de prédicat sémantique, argument d'un prédicat logique et actant d'un prédicat sémantique, fonction propositionnelle et forme propositionnelle représentant la structure actancielle d'un prédicat sémantique. Attention, cependant, il ne s'agit là que d'une métaphore commode, comme celle de valence syntaxique (sur le modèle de la valence atomique). La notion de prédicat sémantique n'est pas une notion logique et les lois de la logique formelle ne s'appliquent que de façon «métaphorique » sur les structures sémantiques prédicatives que manipule la sémantique linguistique telle que nous la concevons.

6 Aux sens liants, c'est-à-dire prédicatifs, on oppose naturellement les sens non liants. Un sens non liant dénote une entité conçue de façon autonome, sans référence à un fait spécifique, et donc ne présuppose pas de participant. Un sémantème correspondant à un sens non liant est appelé nom sémantique. Un nom sémantique peut désigner un objet naturel, une substance, un être, un nombre, un lieu, etc.

7 L'opposition prédicat $\sim$ nom sémantique est donc fondée sur la conjonction de deux propriétés :

- une propriété sémantique générale - dénoter un fait une entité ;

- une propriété de combinatoire - contrôler ne pas contrôler un nombre donné d'actants.

Illustrons ces notions en commençant par les prédicats :

- 'X blesse Y à Z avec W' [Louise a blessé Lulu à la jambe avec un couteau]

- 'pourparlers entre $\mathrm{X}$ et $\mathrm{Y}$ à propos de $\mathrm{Z}$ ' [pourparlers entre le gouvernement et l'opposition à propos de la loi sur l'héritage]

- 'X fidèle à Y' [Louise est fidèle à Lulu]

- 'X rapidement' [écrire rapidement]

- ' $\mathrm{X}$ entre $\mathrm{Y}$ et $\mathrm{Z}$ ' [une ville entre le lac et la montagne]

- ‘À bas Y !’ [À bas la malbouffe!]

On notera qu'un sens prédicatif peut être exprimé par une lexie de n'importe quelle partie du discours.

Pour illustrer les noms sémantiques, citons les sémantèmes 'Balzac', 'Canada', 'soleil', 'sable', 'écureuil', 'soixante-neuf(le nom du nombre 69), etc. Tous ces sémantèmes dénotent des entités et ne contrôlent pas l'expression d'actants sémantiques; du point de vue grammatical, ils ne sont lexicalisés que par des noms au sens large : noms communs, noms propres, pronoms, numéraux. Remarquons que le nom propre - considéré dans les contextes d'emploi élémentaires du type Il s'appelle Balzac, Le nom de ce pays est le Canada, etc. - est pour nous le prototype du nom sémantique, c'est-à-dire du signifié qui ne contrôle aucun actant. Nous ne pouvons malheureusement pas entrer ici dans la discussion de ce délicat problème.

11 La classification en prédicats et noms sémantiques est très claire et ne semble pas poser de problème particulier. Pourtant, elle n'est pas exhaustive et laisse de côté un nombre considérable de sens, car les deux propriétés qui opposent les prédicats aux noms sémantiques sont logiquement indépendantes.

En effet, premièrement, un sens peut dénoter un fait, mais ne pas être liant ; il s'agit d'un prédicat non actanciel - un raccourci pour dire qu'il ne contrôle pas de structure actancielle; on peut citer comme exemples TONNER [En septembre, s'il tonne, la vendange est bonne.], all. BLITZEN 'y avoir un/des éclair(s)' ou russe 'SVETAT' 'commencer à faire jour'. 

que l'on appellera un quasi-prédicat - par exemple, DIRECTEUR ' $X$, le directeur de $Y$ ', ou MAIRIE 'mairie de l'agglomération $Y$ ', ou encore pilule 'pilule qu'avale l'individu $\mathrm{X}$ pour agir sur son état physique $Y$ '.

ous allons examiner ces deux types de sens « spéciaux » à tour de rôle, en concentrant avant tout notre étude sur les quasi-prédicats. Cependant, avant de plonger dans le vif du sujet, nous devons préparer le terrain en approfondissant la notion d'actant sémantique. En effet, ce qui caractérise notre approche de la notion de prédicat est le fait de considérer le prédicat sémantique autant du point de vue de son expression dans la phrase que du point de vue de l'expression de ses actants. À la différence des autres approches, nous insistons donc beaucoup plus sur la facette linguistique (lexicale et syntaxique) que sur la facette logique de la notion.

\section{La notion d'actant sémantique} exprimable dans la phrase sous le contrôle syntaxique de L; cela signifie que l'on peut construire des phrases où l'expression d'un tel sémantème apparait soit comme un dépendant syntaxique direct de L, soit comme un dépendant syntaxique d'un collocatif verbal de L.

21 Il est aisé de concevoir l'« exprimabilité » des positions actancielles dans le cas des prédicats verbaux; celle-ci se manifeste essentiellement par les différents types de dépendants régis par le verbe (le sujet et les compléments). Dans le cas des noms, il faut aussi considérer d'autres types de structures, notamment les collocations verbales (verbes supports et verbes de réalisation), qui permettent d'exprimer les actants auprès des noms prédicatifs, au sein de structures semi-phraséologiques que ces derniers contrôlent. Prenons le cas de ultimatum 'ultimatum communiqué par la personne $\mathrm{X}$ à la personne $\mathrm{Y}$ pour demander l'accomplissement de $\mathrm{Z}$ avant le moment $\mathrm{T}$ '. La collocation à verbe support lancer un ultimatum illustre l'expression de l'actant $\mathrm{X}$ du prédicat nominal auprès de ce dernier: $\mathrm{X}$ est le sujet du verbe support LANCER, dont ULTIMATUM est le complément d'objet. Notons que les structures collocationnelles de ce type sont modélisées dans la Lexicologie Explicative et Combinatoire au moyen du système des fonctions lexicales - voir Mel'čuk (2003a, b). Dans ce cas précis, la collocation en question met en jeu la fonction lexicale de verbe support Oper $_{1}$. 
Pour illustrer l'importance qu'il faut attacher à la seconde condition associée ci-dessus à la position actancielle - exprimabilité (dans la phrase auprès de la lexie prédicative) du sémantème remplissant une position actancielle - comparons deux lexies sémantiquement très proches : ACHETER [Il a acheté ce livre pour 120 \$.] et COUTER [Ce livre lui a couté 120 \$.]. Ces deux lexies permettent de dénoter des situations identiques, où un individu $\mathrm{X}$ obtient une chose $\mathrm{Y}$ auprès d'un autre individu $\mathrm{Z}$ en échange d'une somme d'argent $\mathrm{W}$. On peut considérer que les deux sens en question dénotent des situations impliquant le même nombre de participants (quatre participants au total). Pourtant, alors que ACHETER permet l'expression directe, en tant que compléments, des sémantèmes exprimant les quatre participants en question - Patrick $_{\mathrm{X}}$ a acheté ce livre ${ }_{\mathrm{Y}}$, de Jean-Yves ${ }_{\mathrm{Z}}$, pour $120 \$_{\mathrm{W}^{*}}-$, COÛTER ne permet l'expression que de trois d'entre eux - Ce livre $\mathrm{Y}_{\mathrm{Y}}$ a couté $120 \$_{\mathrm{w}} \grave{a}$ Patrick $\mathrm{x}_{\mathrm{x}}$. Le premier verbe est donc sémantiquement un prédicat contrôlant quatre positions actancielles, alors que le second est un prédicat n'en contrôlant que trois. On voit que la nature prédicative d'un sens, bien que liée directement à la dénotation $d u$ sens en question, est tout de même une propriété fondamentalement linguistique, partiellement indépendante de la dénotation.

Les notions d'actant et de position actancielle, présentées ici dans une perspective presque exclusivement sémantique, doivent en fait être considérées aussi sous l'angle des structures syntaxiques contrôlées par les lexies. Dans la théorie complète des actants présentée dans Mel'čuk (2004), on établit notamment une distinction entre actants sémantiques, syntaxiques profonds et syntaxiques de surface; cependant, nous n'aborderons pas cette distinction ici. Un autre sujet dont nous ne pouvons traiter, mais qui revêt une importance primordiale dans le fonctionnement de la langue, est la hiérarchie des actants sémantiques, en particulier le rôle très spécial du premier actant sémantique.

Nous passons maintenant à l'examen de prédicats non actanciels - pour mieux illustrer le décalage entre les deux aspects qui nous intéressent: la nature sémantique prédicative d'un sens et la présence des positions actancielles qu'il contrôle.

\section{Prédicats non actanciels}

Considérons le sens du verbe PLEUVoIR : le sémantème 'pleuvoir' dénote un fait (plus précisément, un phénomène météorologique), mais il n'appelle pas d'actant! Cette propriété sémantique se manifeste, à la surface, par le fait que PLEUvorr prend comme sujet syntaxique le pronom impersonnel IL, sémantiquement vide ; on le classifie dans les dictionnaires comme verbe impersonnel. Un prédicat sans position actancielle, n'est-ce pas une contradictio in adjecto? Non, puisque le paradoxe n'est qu'apparent. En fait, Il pleut signifie à peu près 'De l'eau tombe du ciel'; or, le prédicat 'tomber', enchâssé dans 'pleuvoir', appelle bel et bien deux positions actancielles, occupées par ses deux actants 'eau' et 'ciel' au sein de la définition de 'pleuvoir'. On peut donc considérer que 'pleuvoir' possède deux positions actancielles déjà saturées, occupées par les deux sémantèmes en question. Nous admettrons ainsi l'existence de ce que nous appellerons des prédicats non actanciels (même si cette appellation est un peu abusive). Un prédicat non actanciel $\mathrm{P}$ contrôle de façon virtuelle au moins un actant sémantique, constant et « incorporé » : un actant d'un prédicat P' se trouvant dans la décomposition sémantique de P. Les langues possèdent un nombre non négligeable de prédicats non actanciels, qui sont les verbes 
météorologiques et assimilables: 'geler' [Il gèle dehors], 'faire beau', 'faire noir', 'faire froid', 'faire soleil', 'être tard' [Il est déjà tard], angl. '[to] dawn' = 'se faire jour' [It was dawning, and the city under us was slowly waking up], russe 'temnet" = 'se faire nuit' et 'mesti' = '[le vent] souffler la neige' ou aloutor 'amtank万' = 'l fait moustiques'.

Les prédicats non actanciels représentent bien entendu un cas très particulier de prédicats, mais ils ne posent pas de problèmes théoriques vis-à-vis de la notion de prédicat. Passons maintenant au cas beaucoup plus délicat des quasi-prédicats.

\section{Quasi-prédicats}

L'essentiel de notre tâche a jusqu'à présent été de préciser des notions connues, adoptées de façon relativement consensuelle par les sémanticiens et lexicologues (prédicat, actant, etc.). Nous allons maintenant aborder ce que l'on peut considérer comme étant un apport original de l'approche Sens-Texte pour ce qui est de la prédicativité : la notion de quasiprédicat.

\section{Quasi-prédicat vs prédicat au sens strict}

Les prédicats que nous avons examinés dans la section précédente - prédicats aux positions actancielles instanciées par des constantes sémantiques inhérentes ('pleuvoir' = 'eau tomber du ciel') - sont atypiques, mais on peut quand même les considérer comme étant simplement des cas extrêmes. En revanche, un quasi-prédicat, qui est un sens liant dénotant une entité est véritablement paradoxal. Comme le terme l'indique, un quasiprédicat n'est pas un prédicat au sens strict, car il ne satisfait pas la propriété sémantique définitoire des prédicats : celle de dénoter un fait. Cependant, l'entité dénotée par un quasi-prédicat est particulière, en ce sens qu'elle est définie plutôt par son implication dans un fait que par ses propriétés intrinsèques; à cause de cela, un quasi-prédicat contrôle des positions actancielles. Par exemple, 'professeur', qui dénote une entité (un individu), dénote avant tout une entité impliquée dans une situation particulière: un individu $\mathrm{X}$ qui enseigne $\mathrm{Y}$ à des individus $\mathrm{Z}$ (dans une institution $\mathrm{W}$ ). Ou encore, 'restaurant'dénote plus qu'un lieu: ce sens dénote un établissement où des individus $X$ servent des individus $Y$ en leur offrant de la nourriture Z. Pour cette raison, les sens de ce type, sans être des prédicats, doivent être considérés comme contrôlant des positions actancielles sémantiques. Un quasi-prédicat est donc un sens liant en bonne et due forme, mais, pour ainsi dire, par ricochet.

Les actants d'un quasi-prédicat sont les actants d'un prédicat véritable « interne », qui apparait au sein du sens en question sans en être le composant central.

Il est essentiel de remarquer que les quasi-prédicats sont toujours lexicalisés, tout comme les noms sémantiques, par des noms.

Le terme quasi-prédicat implique que, dans certaines circonstances, on peut ignorer le fait que le sens en question dénote une entité et le traiter de la même façon qu'on traite les prédicats à part entière. Conséquemment, nous utilisons généralement le terme prédicat pour désigner tous les sens liants, y compris les quasi-prédicats. Cet apparent abus de langage se justifie par le fait qu'il est méthodologiquement nécessaire d'appliquer les mêmes stratégies lexicographiques à tous les sens liants, quels qu'ils soient, à savoir : 1) rédiger une définition introduite par une forme propositionnelle et comprenant des 
variables actancielles, 2) spécifier un schéma de régime explicitant l'expression des actants, avec toutes les contraintes associées, et 3) énumérer les fonctions lexicales relatives aux actants $\left(\mathbf{S}_{\mathrm{i}}, \mathbf{A}_{\mathrm{i}}, \mathbf{A b l e}_{\mathrm{i}}\right.$, Oper $_{\mathrm{i}}$, Func $_{\mathrm{i}}$, Real $_{\mathrm{i}}$, etc.).

\section{Quelques types représentatifs de quasi-prédicats}

Nous ne sommes pas en mesure d'offrir une typologie systématique des quasi-prédicats. Cependant, en nous fondant sur les données du DiCo/LAF, nous présenterons ci-dessous 12 types de quasi-prédicats fréquemment rencontrés dans le travail lexicographique.

Pour chaque type, nous donnerons une formule caractérisant le sens des quasi-prédicats correspondants; dans cette formule, la variable $\mathrm{P}$ désigne le prédicat interne, enchâssé dans le sens quasi-prédicatif considéré, qui donne à ce sens sa nature partiellement prédicative.

1) 'Individu que le locuteur évalue comme P'

IDIOT, GÉNIE : Son mari ${ }_{\mathrm{x}}$ est un idiot, un génie.

Ces sens sont très proches des prédicats véritables. Une de leurs caractéristiques saillantes en français est de s'employer dans la construction ton idiot, génie de mari. Il s'agit de prédicats "de jugement subjectif », qui expriment un point de vue, une opinion du locuteur sur quelque chose.

2) 'Individu qui possède la propriété $P$ '

[un] FRANÇAIS, GAUCHER : Son mari ${ }_{\mathrm{x}}$ est un Français, un gaucher.

Cette classe, contrairement à la précédente, réunit des prédicats «de caractérisation objective ", qui expriment une propriété que le locuteur attribue à quelque chose.

3) 'Individu qui est dans la relation $P$ avec un autre'

MÈRE, VOISINE : Marie ${ }_{\mathrm{X}}$ est la mère, la voisine de Paul $_{\mathrm{Y}}$.

Les quasi-prédicats des classes 1 à 3 entretiennent des relations privilégiées avec les adjectifs : la plupart des noms donnés ci-dessus sont en fait des adjectifs substantivés ( IDIOT $\sim$ [un] IDIOT, FRANÇAIS [un] FRANÇAIS).

4) 'Individu qui fait ou a fait une action P'

SAUVEUR: Hans $_{\mathrm{X}}$ est notre sauveur. $_{\mathrm{Y}}$

ASSASSIN : Princip ${ }_{\mathrm{X}}$ est l'assassin de l'Archiduc ${ }_{\mathrm{Y}}$.

On a ici des noms quasi-prédicatifs dont le contenu peut généralement s'exprimer aussi sous forme de lexies verbales: $X$ qui a sauvé, assassiné $Y$.

5) 'Individu qui a une activité $P$ '

MARCHEUR : Léo est un marcheur fanatique.

MINISTRE : Le ministre français ${ }_{\mathrm{Z}}$ des $_{\text {finances }}$ Gaétan Donnadieu $_{\mathrm{X}}$ a visité Moscou.

Notons que les quasi-prédicats des types 1-5 admettent tous très naturellement un emploi avec copule : Jean $n_{\mathrm{X}}$ est un génie, un professeur ; Jean ${ }_{\mathrm{X}}$ semble être l'ami, l'assassin de Paul $_{\mathrm{Y}} ;$ Jean $_{\mathrm{X}}$ est devenu un marcheur fanatique. Par contre, les quasi-prédicats des types 6-12, qui sont moins prédicatifs, ne sont pas facilement utilisables dans de telles constructions.

6) 'Ensemble d'individus que réunit une activité commune P'

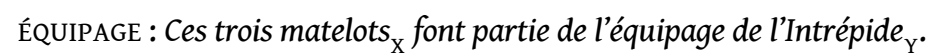


CABINET : Tous les ministres ${ }_{\mathrm{X}}$ du cabinet Brindavoine $e_{\mathrm{Y}}$ ont démissionné.

7) 'Moyen de transport que quelqu'un fait fonctionner $\left[=\mathrm{P}_{1}\right]$ pour transporter $\left[=\mathrm{P}_{2}\right]$ quelque chose'

AUTOCAR : Lulu I $_{\mathrm{Y}}$ pris l'autocar Paris -Rouen $_{\mathrm{W}}$ à cinq heures.

FERRY : L'équipage ${ }_{\mathrm{X}} d u$ ferry de Douvres ${ }_{\mathrm{W}}$ est au complet.

Tout nom d'artefact (c'est-à-dire, d'objet fabriqué ou transformé pour une utilisation particulière) contrôle nécessairement une première position actancielle (X) correspondant à celui qui fait fonctionner ou qui utilise activement l'artefact en question. Un nom d'artefact peut aussi contrôler d'autres positions actancielles, notamment une seconde position $(\mathrm{Y})$ pour les entités auxquelles l'artefact est appliqué.

8) 'Instrument utilisé par quelqu'un pour faire P'

MARTEAU : Marc ${ }_{\mathrm{X}}$ a enfoncé le clou $_{\mathrm{Y}}$ d'un seul coup de marteau.

ÉPÉE : Le marquis ${ }_{\mathrm{X}}$ 'est battu à l'épée avec le mari ${ }_{\mathrm{Y}}$ de sa maitresse.

9) 'Établissement où quelqu'un s'occupe $[=P]$ de quelqu'un d'autre'

CLINIQUE : La jeune femme est finalement sortie de la clinique de soins ${ }_{\mathrm{Z}}$ postnataux.

RESTAURANT : Le restaurant de saucisses de $_{\mathrm{Z}}$ Patrick $_{\mathrm{X}}$ a perdu une partie de sa clientèle ${ }_{\mathrm{Y}}$.

10) 'Animal domestique qui appartient [= P] à quelqu'un'

СНАT : Son $_{\mathrm{x}}$ chat a encore tué un oiseau.

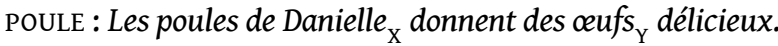

11) 'Partie [= P] de quelque chose'

MORCEAU, MIETTE : morceau, miette de painX

ESTOMAC, JAMBE : Mon $_{\mathrm{x}}$ estomac, $m a_{\mathrm{X}}$ jambe $\mathrm{me}_{\mathrm{X}}$ fait mal.

ACCOUDOIR : -Ne t'assieds pas sur l'accoudoir du fauteuil ${ }_{\mathrm{x}}$.

12) 'ensemble, quantité $[=\mathrm{P}]$ de quelque chose'

FOULE : Une foule d'étudiants $\mathrm{x}_{\mathrm{x}}$ 'est amassée sur la place.

TAS : tas de briques ${ }_{\mathrm{X}}$, de sable $\mathrm{x}_{\mathrm{X}}$

CUILLERÉE : cuillerée de rhum ${ }_{\mathrm{x}}$.

La frontière entre les prédicats, les quasi-prédicats et les noms sémantiques est bien délimitée et il n'y a pas de cas intermédiaires. Cependant, le concept de quasi-prédicat est, lui, gradué. On ressent intuitivement que 'génie' est plus proche d'un prédicat véritable que 'accoudoir', qui lui-même l'est plus que 'chat'. On peut mentionner au moins deux faits linguistiques qui expliquent cette intuition.

Premièrement, on peut difficilement parler «de façon absolue» d'un génie sans conceptualiser un individu $\mathrm{X}$ qui a cette qualité, et d'un accoudoir sans conceptualiser un fauteuil $\mathrm{X}$ dont il est une partie. Il en va tout autrement pour le chat : on peut parler d'un chat sans même penser au fait qu'il a, n'a pas, n'a plus de maitre X.

Deuxièmement, la construction à copule (prédicative par excellence) Quelque chose est $P$ est la façon la plus normale d'utiliser GÉNIE: Quelqu'un est un génie. En revanche, elle est beaucoup moins naturellement compatible avec ACCOUDOIR sans contexte pragmatique fort justifiant cet usage : Quelque chose est un accoudoir. La lexie AccouDoIR, contrairement 
à GÉNIE, est plutôt «faite" pour fonctionner comme actant d'un prédicat véritable : Quelqu'un s'appuie sur un accoudoir.

Les observations qui précèdent nous permettent d'établir la hiérarchie de prédicativité suivante : 'génie' > 'accoudoir' > 'chat'. Cette hiérarchie est conditionnée par la structure interne des sémantèmes correspondants - plus précisément, par le " poids sémantique " et la position du composant prédicatif autour duquel gravite le sémantème en question.

Une meilleure caractérisation de la prédicativité vise notamment à donner au lexicographe des outils conceptuels et descriptifs lui permettant 1) de mieux distinguer les différents types de lexies du point de vue de leur prédicativité, et 2) de mieux standardiser la description des lexies d'un même type prédicatif. Une bonne illustration peut être trouvée dans le cas du vocable polysémique TAUPE. Sa structure peut être décrite de la façon suivante, si l'on choisit de numéroter les acceptions selon l'accroissement de la prédicativité (c'est-à-dire l'ordre inverse de présentation des types de quasi-prédicats, dans la section ci-dessus) :

- TAUPE I [nom sémantique] 'petit animal à fourrure qui vit sous la terre...'

- TAUPE II [quasi-prédicat sémantique 'artefact', proche du cas 8, de la sous-section précédente] 'engin de forage souterrain utilisé par X pour creuser les tunnels Y...'

- TAUPE III [quasi-prédicat sémantique 'individu qui a une activité', proche du cas 5)] 'individu $\mathrm{X}$ qui agit clandestinement dans le milieu $\mathrm{Y}$ pour l'organisation $\mathrm{Z}$ afin de récupérer les informations W...'

Identifier correctement la structure actancielle des quasi-prédicats nous oblige à tenir compte de la cooccurrence restreinte syntaxique et lexicale des lexies en question. Ainsi, pour TAUPE II, il faudra décrire dans son article de dictionnaire les expressions suivantes : La taupe creuse, excave, fore $Y$; pour TAUPE III : $X$ fait la taupe dans $Y, Z$ infiltre/place une taupe dans $Y$, taupe active, taupe dormante, etc.

81 Faute de place, nous ne pouvons malheureusement approfondir ici l'incidence qu'a la prédicativité du sens sur la modélisation lexicographique des lexies correspondantes. On trouvera des informations à ce sujet dans Mel'čuk et al. (1995) et Mel'čuk et Polguère (2006). Les descriptions lexicographiques du français, quant à elles, sont disponibles dans Mel'čuk et al. (1984, 1988, 1992, 1999) et Mel'čuk et Polguère (2007), ainsi que dans les données de la base DiCo accessibles en ligne (http://www.olst.umontreal.ca/dicouebe).

Nous remercions pour leurs commentaires Lidija Iordanskaja, Jasmina Miličevič, Iva Novakova, Ophélie Tremblay ainsi que deux relecteurs anonymes de la revue Lidil. Les travaux de recherche en sémantique lexicale menés à l'observatoire de linguistique Sens-Texte (OLST) sont financés par le Fonds québécois de la recherche sur la société et la culture (FQRSC) et par le Conseil de recherches en sciences humaines du Canada (CRSH).

\section{BIBLIOGRAPHIE}

MEL'ČUK, I. (2003a) : Collocations : définition, rôle et utilité, in F. Grossmann et A. Tutin (dir.), Les collocations : analyse et traitement, Amsterdam, De Werelt, 23-31. 
MEL'ČUK, I. (2003b) : Collocations dans le dictionnaire, in T. Szende (dir.), Les écarts culturels dans les dictionnaires bilingues, Paris, Champion, 19-64.

MEL'ČUK, I. (2004) : Actants in Semantics and Syntax. I. II, Linguistics, $42: 1,1-66$ and $42: 2,247-291$.

MEL'ČUK, I., CLAS, A. et POLGUÈRE, A. (1995) : Introduction à la lexicologie explicative et combinatoire,

Montréal, Les Presses de l'université de Montréal.

MEL'ČUK, I. et POLGUÈRE, A. (2006) : Dérivations sémantiques et collocations dans le DiCo/LAF, Langue

française, 150, 66-83.

MEL'ČUK, I. et POLGUÈRE, A. (2007) : Lexique actif du français. L'apprentissage du vocabulaire fondé sur 20000 dérivations sémantiques et collocations du français, Louvain-la-Neuve, De Boeck Duculot.

MEL'ČUK, I., avec N. Arbatchewsky-Jumarie, L. Elnitsky, L. Iordanskaja et A. Lessard. (1984) :

Dictionnaire explicatif et combinatoire du français contemporain. Recherches lexico-sémantiques, vol. I, Montréal, Les Presses de l'Université de Montréal.

MEL'ČUK, I., avec N. Arbatchewsky-Jumarie, L. Dagenais, L. Elnitsky, L. Iordanskaja, M.N. Lefebvre et S. Mantha. (1988) : Dictionnaire explicatif et combinatoire du français contemporain. Recherches lexico-sémantiques, vol. II, Montréal, Les Presses de l'université de Montréal.

MEL'ČUK, I., avec N. Arbatchewsky-Jumarie, L. Iordanskaja et S. Mantha. (1992) : Dictionnaire explicatif et combinatoire du français contemporain. Recherches lexico-sémantiques, vol. III, Montréal, Les Presses de l'université de Montréal.

MEL'ČUK, I., avec N. Arbatchewsky-Jumarie, L. Iordanskaja, S. Mantha et A. Polguère. (1999) : Dictionnaire explicatif et combinatoire du français contemporain. Recherches lexico-sémantiques, vol. IV, Montréal, Les Presses de l'université de Montréal.

POLGUÈRE, A. (1992) : Remarques sur les réseaux sémantiques Sens ' Texte, in A. Clas (dir.), Le mot, les mots, les bons mots, Montréal, Les Presses de l'Université de Montréal, 109-148.

POLGUÈRE, A. (1997) : Meaning-Text Semantic Networks as a Formal Language, in L. Wanner (ed.), Recent Trends in Meaning-Text Theory, Amsterdam/Philadelphia, John Benjamins, 1-24.

POLGUÈRE, A. (2000) : Une base de données lexicales du français et ses applications possibles en didactique, Lidil, 21, 75-97.

\section{RÉSUMÉS}

La notion de prédicat sémantique permet de distinguer deux classes de sens lexicaux, ou sémantèmes : 1) les prédicats, qui tous dénotent des faits, au sens le plus large (évènements, actions, activités, états, caractéristiques, relations, etc.), et 2) les noms sémantiques, qui dénotent des entités au sens large (êtres vivants, objets physiques, substances, etc.). Nous nous intéressons tout particulièrement au fait qu'il existe une troisième classe de sémantèmes, ni prédicats véritables ni noms sémantiques : il s'agit des quasi-prédicats. Ces derniers dénotent, tout comme les noms sémantiques, des entités et non des faits. Cependant, comme les prédicats, ils ne peuvent être modélisés sans tenir compte de positions actancielles qu'ils contrôlent. L'ensemble des quasi-prédicats d'une langue est très hétérogène, et chaque type de quasi-prédicat pose ses propres problèmes au niveau de la modélisation. Nous examinons différents types de quasiprédicats présents dans les langues, en adoptant une perspective lexicographique. Plus précisément, nous nous situons dans le cadre de la Lexicologie Explicative et Combinatoire, en empruntant nombre de nos illustrations aux données de la base lexicale DiCo des dérivations 
sémantiques et collocations $d u$ français ainsi qu'aux données publiées dans le Lexique actif du français.

The notion of semantic predicate allows for the distinction of two classes of lexical meanings, or semantemes: 1) predicates, all of which denote situations in the broadest sense (events, actions, activities, states, characteristics, relations, etc.), and 2) semantic names, which denote entities, also in the broadest sense (living beings, physical objects, substances, etc.). In this paper we concentrate in particular on the existence of a third class of semantemes that are neither genuine predicates nor semantic names: these are quasi-predicates. Like semantic names, quasipredicates denote entities, and not situations. But like predicates, they cannot be described without accounting for the actant slots they control. The set of quasi-predicates of a language is quite heterogeneous, and each type of quasi-predicate poses its own problems from the point of view of formal description. The paper examines several types of quasi-predicates found in natural languages by putting them into a lexicographic perspective. More precisely, we conduct the discussion in the framework of Explicative and Combinatorial Lexicology, borrowing our illustrations from the lexical database DiCo (semantic derivations and collocations of French) as well as from the recently published Lexique actif du français.

\section{AUTEURS}

\section{IGOR MEL'ČUK}

Université de Montréal, oLST, Département de linguistique et de traduction

\section{ALAIN POLGUÈRE}

Université de Montréal, OLST, Département de linguistique et de traduction 